Pacific Journal of Mathematic 


\title{
ON CONTINUATION OF BOUNDARY VALUES FOR PARTIAL DIFFERENTIAL OPERATORS
}

\author{
H. O. CORDES
}

Let

$$
L=\sum_{i=1}^{n} a_{i}(x) \partial / \partial x_{i}+b(x)
$$

be a first order partial differential operator acting on $m$-component vector functions and defined in a bounded domain $D$ with smooth boundary $\Gamma$. Suppose the $m \times m$-matrices $a_{i}(x)$ are hermitian symmetric and continuously differentiable in $D+\Gamma$. Further let the $m \times m$ matrix $b(x)$ be bounded and measurable over $D+\Gamma$.

Recently K. O. Friedrichs [3] has developed a theory of boundary value problems of the type

$$
\begin{aligned}
(L-\alpha) u & =f, & & x \in D \\
T u & =0, & & x \in \Gamma
\end{aligned}
$$

where $\alpha$ denotes a nonvanishing real constant and $T$ a certain $m \times m$ matrix defined all over the boundary $T$ and satisfying certain further conditions. Concurrently the author worked on the same type of boundary value problem from a different approach extending Friedrich's results to the case of nonlocal boundary conditions [1].

Study of these extensions showed that investigation of the following problem is of basic importance for the author's method:

The question is asked whether a given $m$-component vector function $\varphi$ defined on the boundary $\Gamma$ can be continued into the domain $D$ to become a classical solution $u$ of the equation

$$
L(u)=f
$$

where $f$ is any arbitrary measurable function defined and squared integrable over $D$, which is not given in advance but may be defined after $\varphi$ has been fixed.

Obviously this question is trivially answered "yes" if the boundary and the boundary function are sufficiently smooth. On the other hand if this is not the case, counter examples can be given. It is trivial to find counter examples for special nonelliptic systems but one also can find some for elliptic systems. For instance if the boundary functions $u_{0}, v_{0}$ on the periphery of the unit circle $x^{2}+y^{2}=1$ are defined by

$$
u_{0}=\alpha(\vartheta) \sin \vartheta / 2, \quad v_{0}=-\alpha(\vartheta) \cos \vartheta / 2, \quad 0 \leq \vartheta \leq 2 \pi
$$

Received January 12, 1959. This paper has been prepared under the sponsorship of the Office of Naval Research, Contract No. Nonr 2-228(09). 
and if $\alpha(\vartheta)$ is piecewise continuous and has a jump for any $\vartheta_{0} \neq 0,2 \pi$, then it will be shown in $\S 4$ that there does not exist any couple $u, v$ of real or complex valued functions both being defined and continuously differentiable in the open unit disk $x^{2}+y^{2}<1$ and such that

$$
-u_{x}+v_{y}=f, \quad u_{y}+v_{x}=g
$$

both are squared integrable over $x^{2}+y^{2}<1$;

$$
u, v \text { are uniformly bounded on } x^{2}+y^{2}<1 \text { and }
$$

$$
\begin{aligned}
& \lim _{r \rightarrow 1} u(r \cos \vartheta, r \sin \vartheta)=u_{0}(\vartheta) \\
& \lim _{r \rightarrow 1} v(r \cos \vartheta, r \sin \vartheta)=v_{0}(\vartheta)
\end{aligned}
$$

almost everywhere on $0 \leq \vartheta \leq 2 \pi$.

Considering this problem more carefully it shows that the essential reason for this continuation to be impossible is the following:

The above problem can be connected with the differential operator

$$
L=a_{1} \partial / \partial x+a_{2} \partial / \partial y
$$

with $a_{1}, a_{2}$ being the matrices

$$
a_{1}=\left(\begin{array}{rr}
-1 & 0 \\
0 & 1
\end{array}\right), \quad a_{2}=\left(\begin{array}{ll}
0 & 1 \\
1 & 0
\end{array}\right) .
$$

Using this operator notation we can say that the equation

$$
L \varphi=\psi
$$

with $\varphi, \psi$ being two component vector functions has no classical solution, defined in the unit disk and achieving the boundary values defined by

$$
\varphi(x, y)=\left(u_{0}(\vartheta), v_{0}(\vartheta)\right) \quad x=\cos \vartheta, y=\sin \vartheta
$$

in the sense of the conditions (a), (b), and (c) mentioned above.

If we define

$$
\begin{aligned}
& A(\vartheta)=a_{1} \cos \vartheta+a_{2} \sin \vartheta \\
& \widetilde{A}(\vartheta)=a_{2} \cos \vartheta-a_{1} \sin \vartheta
\end{aligned}
$$

then

$$
L=A(\vartheta) \partial / \partial r+r^{-1} \tilde{A}(\vartheta) \partial / \partial \vartheta .
$$

Hence $A(\vartheta)$ is the coefficient of the derivative in the direction normal to the boundary.

We note that $A(\vartheta)$ is a non-singular (even orthogonal) matrix for 
every $\vartheta$. It will follow from our development that this is the reason why a continuation of discontinuous boundary values becomes impossible. If for some more general operator $L$ the matrix which corresponds to $A(\vartheta)$ is singular on a point or on a set of points then this set can be allowed to contain discontinuities of certain types. And conversely it will be our main result that if $\varphi_{0}$ is bounded measurable only at the boundary and if in addition $A \varphi_{0}$ is Lipschitz continuous then a continuation in the above sense is possible.

The main result is stated in Theorem 3.1. Essentially we will obtain the continuation by use of the elementary solution of the parabolic equation

$$
\nabla^{2} u=\partial u / \partial t
$$

We shall use this for a kind of mollifier. In $\S \S 1$ and 2 we prove some auxiliary results most of which will be known. In order to keep the paper as self contained as possible most of the facts required have been proved explicitely.

1. Auxiliary results. In this section we will establish some known results which have to be used essentially in the following. Let

$$
s^{2}=s_{1}^{2}+s_{2}^{2}+\cdots+s_{p}^{2}
$$

and let the function

$$
\Phi(s ; t)=\Phi\left(s_{1}, \cdots, s_{p} ; t\right)
$$

be defined by

$$
\Phi(s ; t)=(4 \pi t)^{-p / 2} \exp \left(-|s|^{2} / 4 t\right) .
$$

It is known that this function $\Phi(s ; t)$ is the elementary solution of the parabolic equation

$$
\nabla^{2} u=\sum_{i=1}^{p} \partial^{2} u / \partial s_{i}^{2}=\partial u / \partial t
$$

First we note

Lemma 1.1.

$$
\int|s|^{2 k} e-|s|^{2 k} e^{-|s|^{2}} d s=2^{-k} \pi^{-p / 2} p(p+2)(p+4) \cdots(p+2 k-2) .
$$

Here the integral extends over the whole $\left(s_{1}, \cdots, s_{p}\right)$-space.

The proof of Lemma 1.1 can be obtained by repeated application of Green's formula. 


$$
f(s)=f\left(s_{1}, \cdots, s_{p}\right)
$$

be a (scalar) complex valued bounded measurable function defined and nonnegative for

$$
-\infty<s_{j}<\infty, \quad j=1, \cdots, p .
$$

Let $s_{0}$ be any point and let $\triangle$ denote the cube

$$
\left|s_{j}-s_{j}^{0}\right| \leq \delta, \quad j=1, \cdots, p .
$$

Statement. If

$$
\lim _{\delta \rightarrow 0} \delta^{-p} \int_{\Delta} f(s) d s=0
$$

then

$$
\lim _{t \rightarrow 0} \int_{\Delta} \Phi\left(s_{0}-s^{\prime} ; t\right) f\left(s^{\prime}\right) d s^{\prime}=0
$$

the integral in (1.10) being taken over the whole $s$-space.

Proof. It is obvious that we can restrict ourself to the case $s_{0}=0$. Now, (1.9) being satisfied, let

$$
\beta\left(\delta_{0}\right)=\sup _{0<\delta \leqq \delta_{0}}\left\{\delta^{-p} \int_{A} f(s) d s\right\}^{(p+1)^{-1}}
$$

and let

$$
\gamma(\delta)=\delta(\delta+\beta(\delta))
$$

$\gamma(\delta)$ is a strictly monotonically increasing function of $\delta$, and $\gamma(0)=0$. Hence the inverse function $\delta=\delta(\gamma)$ exists in some right neighborhood of $\gamma=0$ and $\delta(0)=0$. Also

$$
\begin{array}{r}
\gamma^{-p} \int_{\Delta} f(s) d s \leqq(\delta+\beta(\delta))^{-p} \beta(\delta)^{p+1} \\
\leq \beta(\delta) \longrightarrow 0, \delta \longrightarrow 0 .
\end{array}
$$

Hence

$$
\lim _{\gamma \rightarrow 0} \gamma^{-p} \int_{\Delta} f(s) d s=0 .
$$

Let

$$
\pi=\delta / \gamma,
$$

then

$$
\lim _{\gamma \rightarrow 0} \tau(\gamma)=\infty
$$


Let $\Delta^{\prime}$ be the cube $\left|s_{j}\right| \leq \gamma, j=1, \cdots, p$. Then by (1.15) $\Delta$ can be written in the form

$$
\Delta=\tau \Delta^{\prime}
$$

and (1.14) reads

$$
\lim _{\gamma \rightarrow 0} \gamma^{-p} \int_{\tau d^{\prime}} f(s) d s=0 .
$$

Now for any given $t>0$ set $\gamma=t^{1 / 2}$, then

$$
\int \Phi\left(s_{0}-s^{\prime} ; t\right) f\left(s^{\prime}\right) d s^{\prime}=\int \Phi(s ; t) f(s) d s=\int_{\tau d^{\prime}}+\int_{C\left(\tau d^{\prime}\right)}
$$

where $C\left(\tau \Delta^{\prime}\right)$ denotes the complement of the cube $\tau \Delta^{\prime}$ with respect to the whole $s$-space. But remembering the definition of $\Phi(s ; t)$ we obtain for the first integral

$$
\leq(4 \pi)^{-p / 2} \gamma^{-p} \int_{\tau d^{\prime}} f(s) d s
$$

and hence for $t \rightarrow 0$, i.e., $\gamma \rightarrow 0$ the first integral tends to zero by (1.18). On the other hand $f(s)$ is assumed to be uniformly bounded, hence the second integral can be estimated by

$$
\begin{gathered}
c_{0} \int_{C(\tau A l)} \Phi(s ; t) d s \\
\leqq c_{0}(4 \pi)^{-p / 2} \gamma^{-p}\left\{\int_{|\sigma| \geqq \tau \gamma} e^{-\sigma^{2} / \gamma^{2}} d \sigma\right\}^{p} \\
=c_{0}(4 \pi)^{-p / 2}\left\{\int_{|\sigma| \geqq \tau} e^{-\sigma^{2}} d \sigma\right\}^{p} .
\end{gathered}
$$

But by (1.16)

$$
\pi=\tau(\gamma)=\tau\left(t^{1 / 2}\right)
$$

tends to $\infty$ at $t \rightarrow 0$. Therefore the second integral also tends to zero. This proves the lemma.

Lemma 1.3. Let $\Phi(s ; t)$ be as defined in (1.3) and let

$$
\Psi_{i}(s ; t)=\partial / \partial s_{i} \Phi(s ; t) .
$$

Then

$$
\begin{aligned}
& \int d s \Phi\left(s-s^{\prime} ; t\right) \Phi\left(s-s^{\prime \prime} ; t\right)=(8 \pi t)^{-p / 2} \exp \left(-\left|s^{\prime}-s^{\prime \prime}\right|^{2} / 8 t\right) \\
& \quad \int d s \Psi_{i}\left(s-s^{\prime} ; t\right) \Psi_{i}\left(s-s^{\prime \prime} ; t\right) \\
& =(4 t)^{-1}(8 \pi t)^{-p / 2}\left(1-\left(s_{i}^{\prime}-s_{i}^{\prime \prime}\right)^{2} / 4 t\right) \exp \left(-\left|s^{\prime}-s^{\prime \prime}\right|^{2} / 8 t\right)
\end{aligned}
$$


both integrals being taken over the whole $\left(s_{1}, \cdots, s_{p}\right)$-space.

Proof. We only remark that

$$
\begin{aligned}
& \exp \left(-\left|s-s^{\prime}\right|^{2} / 4 t\right) \exp \left(-\left|s-s^{\prime \prime}\right|^{2} / 4 t\right) \\
= & \exp \left(-\left|s^{\prime}-s^{\prime \prime}\right|^{2} 8 t\right) \exp \left(-|\hat{s}|^{2} / 2 t\right)
\end{aligned}
$$

where we denote

$$
\hat{s}=s-1 / 2\left(s^{\prime}+s^{\prime \prime}\right) .
$$

Therefore the integral (1.24) equals to

$$
(4 \pi t)^{-p} \exp \left(-\left|s^{\prime}-s^{\prime \prime}\right|^{2} / 8 t\right) \int \exp \left(-|\hat{s}|^{2} / 2 t\right) d \hat{s}
$$

and clearly

$$
\int \exp \left(-|\hat{s}|^{2} / 2 t\right) d \hat{s}=(2 \pi t)^{p / 2} .
$$

This proves the first formula. For the second formula we note that

$$
\Psi_{i}(s ; t)=-(2 t)^{-1}(4 \pi t)^{-p / 2} s_{i} \exp \left(-|s|^{2} / 4 t\right) .
$$

Now

$$
\left(s_{i}-s_{i}^{\prime}\right)\left(s_{i}-s_{i}^{\prime \prime}\right)=\hat{s}_{i}^{2}-1 / 4\left(s_{i}^{\prime}-s_{i}^{\prime \prime}\right)^{2} .
$$

Hence the integral (1.25) gets the form

$$
\begin{aligned}
& (2 t)^{-2}(4 \pi t)^{-p} \exp \left(-\left|s^{\prime}-s^{\prime \prime}\right|^{2} / 8 t\right) \\
& \times\left\{\int \hat{s} \exp \left(-|\hat{s}|^{2} / 2 t\right) d \hat{s}-1 / 4\left(s_{i}^{\prime}-s_{i}^{\prime \prime}\right)^{2} \int \exp \left(-|\hat{s}|^{2} / 2 t\right) d \hat{s}\right\} .
\end{aligned}
$$

But

$$
\int \hat{s}_{i}^{2} \exp \left(-|\hat{s}|^{2} / 2 t\right) d \hat{s}=t(2 \pi t)^{p / 2} .
$$

If we substitute (1.29) and (1.33) into (1.32) then we get

$$
=(4 t)^{-1}(8 \pi t)^{-p / 2}\left(1-\left(s_{i}^{\prime}-s_{i}^{\prime \prime}\right)^{2} / 4 t\right) \exp \left(-\left|s^{\prime}-s^{\prime \prime}\right|^{2} / 8 t\right)
$$

which completes the proof.

Lemma 1.4. Let

$$
\begin{aligned}
& \Omega_{1}(s ; t)=(2 t)^{-1}(4 \pi t)^{-p / 2} \exp \left(-|s|^{2} / 4 t\right) \\
& \Omega_{2}(s ; t)=|s|^{2}(2 t)^{-2}(4 \pi t)^{-p / 2} \exp \left(-|s|^{2} / 4 t\right) .
\end{aligned}
$$

Statement. 
$(1.37)$

$$
\begin{aligned}
& \int d s \Omega_{1}\left(s-s^{\prime} ; t\right) \Omega_{1}\left(s-s^{\prime \prime} ; t\right) \sum_{i=1}^{p}\left(s_{i}-s_{i}^{\prime}\right)\left(s_{i}-s_{i}^{\prime \prime}\right) \\
= & -1 / 2 d / d t\left((8 \pi t)^{-p / 2} \exp \left(-\left|s^{\prime}-s^{\prime \prime}\right|^{2} / 8 t\right)\right) \\
& \int d s \Omega_{2}\left(s-s^{\prime} ; t\right) \Omega_{2}\left(s-s^{\prime \prime} ; t\right) \sum_{i=1}^{p}\left(s_{i}-s_{i}^{\prime}\right)\left(s_{i}-s_{i}^{\prime \prime}\right) \\
= & -1 / 2 d / d t\left[( 8 \pi t ) ^ { - p / 2 } \left\{(8 t)^{-2}\left|s^{\prime}-s^{\prime \prime}\right|^{4}\right.\right. \\
+ & \left.\left.p(8 t)^{-1}\left|s^{\prime}-s^{\prime \prime}\right|^{2}+1 / 4(p+2)(p+4)\right\} \exp \left(-\left|s^{\prime}-s^{\prime \prime}\right|^{2} / 8 t\right)\right] .
\end{aligned}
$$

Proof. We introduce the notation

$$
\hat{\sigma}=(2 t)^{-1 / 2}\left(s-1 / 2\left(s^{\prime}+s^{\prime \prime}\right)\right), \quad \sigma^{*}=(8 t)^{-1 / 2}\left(s^{\prime}-s^{\prime \prime}\right)
$$

and we observe that

$$
\sum_{i=1}^{p}\left(s_{i}-s_{i}^{\prime}\right)\left(s_{i}-s_{i}^{\prime \prime}\right)=2 t\left(|\hat{\sigma}|^{2}-\left|\sigma^{*}\right|^{2}\right) .
$$

Now if we substitute (1.36) and (1.40) into the integral (1.37) this integral equals

$$
\begin{aligned}
& (2 t)^{-1}\left(8 \pi^{2} t\right)^{-p / 2} \exp \left(-\left|\sigma^{*}\right|^{2}\right) \int\left(|\hat{\sigma}|^{2}-\left|\sigma^{*}\right|^{2}\right) \exp \left(-|\hat{\sigma}|^{2} d \hat{\sigma}\right) \\
& =(8 \pi)^{-p / 2}\left(p / 4 t^{-p / 2-1}-1 / 16\left|s^{\prime}-s^{\prime \prime}\right|^{2} t^{-p / 2-2}\right) \exp \left(-\left|s^{\prime}-s^{\prime \prime}\right|^{2} / 8 t\right)
\end{aligned}
$$

Here for the evaluation of

$$
\int|\hat{\sigma}|^{2} \exp \left(-|\hat{\sigma}|^{2}\right) d \hat{\sigma}
$$

Lemma 1.1 has been applied. Now (1.41) is equal to the derivative in (1.37) as can be proved by differentiation. Therefore (1.37) is proved. For the second integral we get in a similar way the expression

$$
\begin{aligned}
& (2 t)^{-1}\left(8 \pi^{2} t\right)^{-p / 2} \exp \left(-\left|\sigma^{*}\right|^{2}\right) \\
& \times \int\left|\hat{\sigma}-\sigma^{*}\right|^{2}\left|\hat{\sigma}+\sigma^{*}\right|^{2}\left(|\hat{\sigma}|^{2}-\left|\sigma^{*}\right|^{2}\right) \exp \left(-|\hat{\sigma}|^{2}\right) d \hat{\sigma} .
\end{aligned}
$$

Here we were using that

$$
s-s^{\prime}=(2 t)^{1 / 2}\left(\hat{\sigma}-\sigma^{*}\right), \quad s-s^{\prime \prime}=(2 t)^{1 / 2}\left(\hat{\sigma}+\sigma^{*}\right) .
$$

We observe that

$$
\left|\hat{\sigma}-\sigma^{*}\right|^{2}\left|\hat{\sigma}+\sigma^{*}\right|^{2}=\left(|\hat{\sigma}|^{2}+\left|\sigma^{*}\right|^{2}\right)^{2}-4\left(\hat{\sigma} \sigma^{*}\right)^{2}
$$

and further that

$$
\int\left(\hat{\sigma} \sigma^{*}\right)^{2}\left(|\hat{\sigma}|^{2}-\left|\sigma^{*}\right|^{2}\right) \exp \left(-|\hat{\sigma}|^{2}\right) d \hat{\sigma}
$$




$$
\begin{aligned}
& =\sum_{i=1}^{p}\left\{\left(\sigma_{i}^{*}\right)^{2} \int\left(\hat{\sigma}_{i}\right)^{2}\left(|\hat{\boldsymbol{\sigma}}|^{2}-\left|\sigma^{*}\right|^{2}\right) \exp \left(-|\hat{\boldsymbol{\sigma}}|^{2}\right) d \hat{\boldsymbol{\sigma}}\right\} \\
& =1 / p\left|\sigma^{*}\right|^{2} \int|\hat{\boldsymbol{\sigma}}|^{2}\left(|\hat{\boldsymbol{\sigma}}|^{2}-\left|\sigma^{*}\right|^{2}\right) \exp \left(-|\hat{\boldsymbol{\sigma}}|^{2}\right) d \hat{\boldsymbol{\sigma}} .
\end{aligned}
$$

Here we used that

$$
\int \hat{\sigma}_{i} \hat{\sigma}_{k}\left(|\hat{\sigma}|^{2}-\left|\sigma^{*}\right|^{2}\right) \exp \left(-|\hat{\sigma}|_{0}^{2}\right) d \hat{\sigma}=0, i \neq k .
$$

Substituting (1.45) and (1.46) into (1.43) we get the expression

$$
\begin{aligned}
& (2 t)^{-1}\left(8 \pi^{2} t\right)^{-p / 2} e^{-\left|\sigma^{*}\right|^{2}} \int\left(|\hat{\sigma}|^{4}\right. \\
& \left.\quad+\left|\sigma^{*}\right|^{4}+2(p-2) / p|\hat{\sigma}|^{2}\left|\sigma^{*}\right|^{2}\right)\left(|\hat{\sigma}|^{2}-\left|\sigma^{*}\right|^{2}\right) e^{-\mid \hat{\sigma}^{2}} d \hat{\sigma} .
\end{aligned}
$$

Further

$$
\begin{gathered}
\left(|\hat{\boldsymbol{\sigma}}|^{4}+\left|\sigma^{*}\right|^{4}+2(p-2) / p|\hat{\boldsymbol{\sigma}}|^{2}\left|\sigma^{*}\right|^{2}\right)\left(|\hat{\boldsymbol{\sigma}}|^{2}-\left|\sigma^{*}\right|^{2}\right) \\
=|\hat{\boldsymbol{\sigma}}|^{6}+(p-4) / p|\hat{\boldsymbol{\sigma}}|^{4}\left|\sigma^{*}\right|^{2}-(p-4) / p|\hat{\boldsymbol{\sigma}}|^{2}\left|\sigma^{*}\right|^{4}-\left|\sigma^{*}\right|^{6} .
\end{gathered}
$$

We substitute this into (1.38) and then use Lemma 1.2 to evaluate the integral, then this integral equals

$$
\begin{aligned}
& \pi^{-p / 2}\{1 / 8 p(p+2)(p+4) \\
& \left.+1 / 4(p+2)(p-4)\left|\sigma^{*}\right|^{2}-1 / 2(p-4)\left|\sigma^{*}\right|^{4}-\left|\sigma^{*}\right|^{6}\right\} .
\end{aligned}
$$

On the other hand by calculating the derivative (1.38) we get the expression

$$
\begin{gathered}
-1 / 2(8 \pi)^{-p / 2}\left\{-1 / 2(p+4) t^{-p / 2-1}\left|\sigma^{*}\right|^{4}-1 / 2 p(p+2) t^{-p / 2-1}\left|\sigma^{*}\right|^{2}\right. \\
\left.-1 / 8 p(p+2)(p+4) t^{-p / 2-1}\right\} \exp \left(-\left|\sigma^{*}\right|^{2}\right) \\
-1 /(2 t)(8 \pi t)^{-p / 2}\left\{\left|\sigma^{*}\right|^{6}+p\left|\sigma^{*}\right|^{4}+1 / 4(p+2)(p+4)\left|\sigma^{*}\right|^{2}\right\} \exp \left(-\left|\sigma^{*}\right|^{2}\right) \\
=-(2 t)^{-1}(8 \pi t)^{-p / 2} \exp \left(-\left|\sigma^{*}\right|^{2}\right)\left\{\left|\sigma^{*}\right|^{6}+1 / 2(p-4)\left|\sigma^{*}\right|^{4}\right. \\
\left.-1 / 4(p+2)(p-4)\left|\sigma^{*}\right|^{2}-1 / 8 p(p+2)(p+4)\right\} .
\end{gathered}
$$

If we substitute (1.50) into (1.49) and then compare the obtained expression with (1.51) we find that both are equal. Therefore formula (1.38) is proved.

2. Lemmata about special integral operators. The following lemma was used earlier by K. O. Friedrichs [2]. It can be considered to be a translation of a theorem about infinite matrices going back to $I$. Schur [6].

LEMMA 2.1. Let

$$
X\left(s ; s^{\prime}\right)=X\left(s_{1}, \cdots, s_{p} ; s_{1}^{\prime}, \cdots, s_{1}^{\prime}, \cdots, s_{p}^{\prime}\right)
$$


be defined and continuous for $s, s^{\prime} \in D_{0}, D_{0}$ being any region of $\left(s_{1}, \cdots, s_{p}\right)$ space, and let

$$
\begin{aligned}
& \gamma=\sup _{s \in D_{0}} \int_{D_{0}}\left|X\left(s ; s^{\prime}\right)\right| d s^{\prime} \\
& \delta=\sup _{t^{\prime} \in D_{0}} \int_{D_{0}}\left|X\left(s ; s^{\prime}\right)\right| d s .
\end{aligned}
$$

Statement.

$$
\int_{D_{0}} d s\left|\int_{D_{0}} X\left(s, s^{\prime}\right) u\left(s^{\prime}\right) d s^{\prime}\right|^{2} \leq \gamma^{\delta} \int_{D_{0}}|u(s)|^{2} d s
$$

holds for every complex valued measurable function $u(s)$ which is squared integrable over $D_{0}$.

Proof. By Schwarz' inequality

$$
\begin{aligned}
& \int_{D_{0}} d s\left|\int_{D_{0}} X\left(s ; s^{\prime}\right) u\left(s^{\prime}\right) d s^{\prime}\right|^{2} \leq \int_{D_{0}} d s\left(\int_{D_{0}}\left|X\left(s ; s^{\prime}\right)\right|\left|u\left(s^{\prime}\right)\right| d s^{\prime}\right)^{2} \\
& \quad \leq \int_{D_{0}} d s\left\{\int_{D_{0}}\left|X\left(s ; s^{\prime}\right)\right| d s^{\prime} \int_{D_{0}}\left|X\left(s ; s^{\prime}\right)\right|\left|u\left(s^{\prime}\right)\right|^{2} d s^{\prime}\right\} \\
& \quad \leq \gamma \int_{D_{0}}\left|u\left(s^{\prime}\right)\right|^{2}\left(\int_{D_{0}}\left|X\left(s ; s^{\prime}\right)\right| d s\right) d s^{\prime} \leq \gamma^{5} \int_{D_{0}}\left|u\left(s^{\prime}\right)\right|^{2} d s^{\prime}
\end{aligned}
$$

Now let $\Phi(s ; t), \Psi_{i}(s ; t) ; \Omega_{1}(s ; t), \Omega_{2}(s ; t)$ be defined as in (1.1), (1.23), (1.35), and (1.36).

\section{LEMMA 2.2.}

$$
\sum_{i=1}^{p} \iint d s d t\left|\int \Psi_{i}\left(s-s^{\prime} ; t\right) u\left(s^{\prime}\right) d s^{\prime}\right|^{2} \leq \int|u(s)|^{2} d s
$$

for every $u(s)$ squared integrable over the whole $s$-space and having a compact carrier. Here the integral $\int d t$ is taken over the interval $0 \leq t \leq 1$, the integrals $\int d s$ and $\int d s^{\prime}$ are considered to be taken over the whole $s$-space.

Proof. First of all by Lemma 1.3:

$$
\begin{aligned}
& \sum_{i=1}^{p} \iint d s d t\left|\int \Psi_{\imath}\left(s-s^{\prime} ; t\right) u\left(s^{\prime}\right) d s^{\prime}\right|^{2} \\
& =\lim _{\varepsilon \rightarrow 0} \iint d s^{\prime} d s^{\prime \prime} \overline{u\left(s^{\prime}\right)} u\left(s^{\prime \prime}\right) \int_{\varepsilon}^{1} d t \sum_{i=1}^{p} \int d s \Psi_{i}\left(s-s^{\prime} ; t\right) \Psi_{i}\left(s-s^{\prime \prime} ; t\right) \\
& =\lim _{\varepsilon \rightarrow 0} \iint d s^{\prime} d s^{\prime \prime} \overline{u\left(s^{\prime}\right)} u\left(s^{\prime \prime}\right) \int_{\varepsilon}^{1} d t(4 t)^{-1}(8 \pi t)^{-p / 2} \\
& \quad \times\left(p-(4 t)^{-1}\left|s^{\prime}-s^{\prime \prime}\right|^{2}\right) \exp \left(-\left|s^{\prime}-s^{\prime \prime}\right|^{2} / 8 t\right)
\end{aligned}
$$


But as we saw in the proof of Lemma 1.4 (formula (1.51)) this integrand is equal to

$$
-1 / 2 d / d t\left\{(8 \pi t)^{-p / 2} \exp \left(-\left|s^{\prime}-s^{\prime \prime}\right|{ }^{2} / 8 t\right)\right\}
$$

and hence the right hand side equals to

$$
\begin{aligned}
= & -1 / 2 \lim _{\varepsilon \rightarrow 0} \iint d s^{\prime} d s^{\prime \prime} \overline{u\left(s^{\prime}\right) u}\left(s^{\prime \prime}\right) \\
& \times\left\{(8 \pi)^{-p / 2} \exp \left(-\left|s^{\prime}-s^{\prime \prime}\right|^{2} / 8\right)-(8 \pi \varepsilon)^{-p / 2} \exp \left(-\left|s^{\prime}-s^{\prime \prime}\right|^{2} / 8\right)\right\} \\
\leq & 1 / 2 \lim _{\varepsilon \rightarrow 0} \int d s^{\prime} \overline{u\left(s^{\prime}\right)} \int d s^{\prime \prime}(8 \pi \varepsilon)^{-p / 2} \exp \left(-\left|s^{\prime}-s^{\prime \prime}\right|^{2} / 8 \varepsilon\right) u\left(s^{\prime \prime}\right) \\
\leq & 1 / 2 \lim _{\varepsilon \rightarrow 0}\left\{\int|u(s)|^{2} d s(8 \pi \varepsilon)^{-p}\right. \\
& \left.\times \int d s^{\prime}\left|\int \exp \left(-\left|s^{\prime}-s^{\prime \prime}\right|^{2} / 8 \varepsilon\right) u\left(s^{\prime \prime}\right) d s^{\prime \prime}\right|^{2}\right\}^{1 / 2} .
\end{aligned}
$$

Here we were using that the kernel $\exp \left(-\left|s^{\prime}-s^{\prime \prime}\right|^{2} / 8\right)$ is positive definite as can be easily seen by Lemma 1.3. Since

$$
\int \exp \left(-\left|s^{\prime}-s^{\prime \prime}\right|^{2} / 8 \varepsilon\right) d s^{\prime}=\int \exp \left(-\left|s^{\prime}-s^{\prime \prime}\right|^{2} / 8 \varepsilon\right) d s=(8 \pi \varepsilon)^{p / 2}
$$

Lemma 2.1 yields

$$
(8 \pi \varepsilon)^{-p} \int d s^{\prime} \mid \int \exp \left(-\left|s^{\prime}-s^{\prime \prime}\right|^{2} / 8 \varepsilon\right) u\left(\left.s^{\prime \prime}\right|^{2} \leq \int|u(s)|^{2} d s .\right.
$$

This completes the proof of Lemma 2.2.

\section{LEMMA 2.3. Let}

$$
\Omega(s ; t)=d / d t \Phi(s ; t)
$$

and let $v(s)$ be Lipschitz continuous over the whole $\left(s_{1}, \cdots, s_{p}\right)$-space and with compact carrier.

\section{Statement.}

$$
\iint d s d t\left|\int d s^{\prime} \Omega\left(s-s^{\prime} ; t\right) v(s)\right|^{2} \leq\left. p \int \sum_{i=1}^{p}|\partial v| \partial s_{i}\right|^{2} d s .
$$

Proof. Since $\Phi(s ; t)$ is a solution of the parabolic equation (1.29) we get

$$
\Omega(s ; t)=\sum_{i=1}^{p} \partial / \partial s_{i} \Psi_{i}(s ; t)
$$

and hence by Green's formula 


$$
\int d s^{\prime} \Omega\left(s-s^{\prime} ; t\right) v\left(s^{\prime}\right)=\sum_{i=1}^{p} \int \Psi_{i}\left(s-s^{\prime} ; t\right) v_{i}\left(s^{\prime}\right) d s^{\prime}
$$

where we denote

$$
v_{i}(s)=\partial / \partial s_{i}(v(s))
$$

Consequently

$$
\begin{aligned}
& \iint d s d t\left|\int d s^{\prime} \Omega\left(s-s^{\prime} ; t\right) v\left(s^{\prime}\right)\right|^{2} \\
& \quad \leq p \sum_{i=1}^{p} \iint d s d t\left|\int d s^{\prime} \Psi_{i}\left(s-s^{\prime} ; t\right) v_{i}\left(s^{\prime}\right)\right|^{2} \\
& \quad \leq p \sum_{i=1}^{p}\left(\sum_{k=1}^{p} \iint d s d t\left|\int d s^{\prime} \Psi_{k}\left(s-s^{\prime} ; t\right) v_{i}\left(s^{\prime}\right)\right|^{2}\right) \\
& \quad \leq p \sum_{i=1}^{p} \int\left|\partial v / \partial s_{i}\right|^{2} d s
\end{aligned}
$$

which prove the lemma.

In the following $c$ always denotes a constant not depending on $u(s)$.

LEMMA 2.4.

$$
\iint d s d t\left|\int d s^{\prime} \Omega\left(s-s^{\prime} ; t\right)\left(s_{i}-s_{i}^{\prime}\right) u\left(s^{\prime}\right)\right|^{2} \leq c \int|u(s)|^{2} d s
$$

for any arbitrary $u(s)$ with compact carrier and squared integrable over the $s$-space.

Proof. Clearly

$$
\begin{aligned}
& \Omega(s ; t)=d / d t \Phi(s ; t) \\
& \quad=(4 \pi t)^{-p / 2}\left(|s|^{2} /(4 t)^{2}-p /(2 t)\right) \exp \left(-|s|^{2} / 4 t\right) \\
& \quad=\Omega_{2}(s ; t)-p \Omega_{1}(s ; t) .
\end{aligned}
$$

Hence the integral in (2.16) can be estimated by

$$
\begin{aligned}
& 2 \sum_{i=1}^{p} \iint d s d t\left|\int d s^{\prime} \Omega_{2}\left(s-s^{\prime} ; t\right)\left(s_{i}-s_{i}^{\prime}\right) u\left(s^{\prime}\right)\right|^{2} \\
& \quad+2 p^{2} \sum_{i=1}^{p} \iint d s d t\left|\int d s^{\prime} \Omega_{1}\left(s-s^{\prime} ; t\right)\left(s_{i}-s_{i}^{\prime}\right) u\left(s^{\prime}\right)\right|^{2} .
\end{aligned}
$$

Now this can be written in the form

$$
\begin{aligned}
& 2 \lim _{\varepsilon \rightarrow 0} \iint d s^{\prime} d s^{\prime \prime} \overline{u\left(s^{\prime}\right)} u\left(s^{\prime \prime}\right) \\
& \quad \times \int_{\varepsilon}^{1} d t \sum_{i=1}^{p} \int d s \Omega_{2}\left(s-s^{\prime} ; t\right) \Omega_{2}\left(s-s^{\prime \prime} ; t\right)\left(s_{i}-s_{i}^{\prime}\right)\left(s_{i}-s_{i}^{\prime \prime}\right) \\
& +2 p^{2} \lim _{\varepsilon \rightarrow 0} \iint d s^{\prime} d s^{\prime \prime} \overline{u\left(s^{\prime}\right)} u\left(s^{\prime \prime}\right)
\end{aligned}
$$




$$
\times \int_{\varepsilon}^{1} d t \sum_{i=1}^{p} \int d s \Omega_{1}\left(s-s^{\prime} ; t\right) \Omega_{1}\left(s-s^{\prime \prime} ; t\right)\left(s_{i}-s_{i}^{\prime}\right)\left(s_{i}-s_{i}^{\prime \prime}\right) .
$$

We apply Lemma 1.4 and this equals

$$
\begin{aligned}
& -\lim _{\varepsilon \rightarrow 0} \int d s^{\prime} d s^{\prime \prime} \overline{u\left(s^{\prime}\right)} u\left(s^{\prime \prime}\right) \\
& \quad \times\left\{(8 \pi)^{-p / 2} \Xi_{1}\left(\left|s-s^{\prime}\right|^{2} / 8\right)-(8 \pi \varepsilon)^{-p / 2} \Xi_{1}\left(\left|s^{\prime}-s^{\prime \prime}\right|^{2} / 8 \varepsilon\right)\right\} \\
& \quad \times \exp \left(-\left|s^{\prime}-s^{\prime \prime}\right|^{2} / 8 \varepsilon\right)
\end{aligned}
$$

where $\Xi_{1}(\alpha)$ means a certain polynomial in $\alpha$ with constant coefficients and of degree two, the coefficients only depending on $p$. By a treatment similar to the last expression of Lemma 2.3 we get the final statement.

LEMMA 2.5 .

$$
\begin{aligned}
& \iint d s d t\left|\int d s^{\prime}\right| \Omega\left(s-s^{\prime} ; t\right)|| s-\left.\left.s^{\prime}\right|^{1+\varepsilon} u\left(s^{\prime}\right)\right|^{2} \\
& \quad \leq c(\varepsilon) \int|u(s)|^{2} d s
\end{aligned}
$$

for any positive $\varepsilon$ and for any arbitrary $u(s)$ with compact carrier and squared integrable over the whole space, $c(\varepsilon)$ being a constant independent of $u(s)$.

Proof. Clearly it suffices to prove the corresponding inequality with $\Omega\left(s-s^{\prime} ; t\right)$ replaced by $\Omega_{j}\left(s-s^{\prime} ; t\right), j=1,2$. In order to achieve these estimates we again use the notation (1.49) and estimate as follows:

$$
\begin{aligned}
\int d s & \left|\Omega_{1}\left(s-s^{\prime} ; t\right)\right|\left|\Omega_{1}\left(s-s^{\prime \prime} ; t\right)\right|\left[\left|s-s^{\prime}\right|^{2}\left|s-s^{\prime \prime}\right|^{2}\right]^{(1+\varepsilon) / 2} \\
= & (2 t)^{-1+\varepsilon}\left(8 \pi^{2} t\right)^{-p / 2} \exp \left(-\left|s^{\prime}-s^{\prime \prime}\right|^{2} / 8 t\right) \int d \hat{\sigma} e^{-|\hat{\mid}|^{2}} \\
& \times\left\{\left(|\hat{\sigma}|^{2}+\left|\sigma^{*}\right|^{2}\right)^{2}-4\left(\hat{\sigma} \sigma^{*}\right)^{2}\right\}^{(1+\varepsilon) / 2} \\
= & (2 t)^{-1+\varepsilon}\left(8 \pi^{2} t\right)^{-p / 2} \exp \left(-\left|s^{\prime}-s^{\prime \prime}\right|^{2} / 8 t\right) J\left(s^{\prime}-\left.s^{\prime \prime}\right|^{2} / 8 t\right)
\end{aligned}
$$

where

$$
\begin{aligned}
& J\left(\left|\sigma^{*}\right|^{2}\right)=\int d \hat{\sigma} e^{-|\hat{\sigma}|^{2}}\left\{\left(|\hat{\sigma}|^{2}+\left|\sigma^{*}\right|^{2}\right)^{2}-4\left(\hat{\sigma} \sigma^{*}\right)^{2}\right\}^{(1+\varepsilon) / 2} \\
& \quad \leq \int d \hat{\sigma} \exp \left(-|\hat{\sigma}|^{2}\right)\left\{|\hat{\sigma}|^{2}+\left|\sigma^{*}\right|^{2}\right\}^{(1+\varepsilon)} \\
& \quad \leq 2^{2} \int d \hat{\sigma} \exp \left(-|\hat{\sigma}|^{2}\right)|\hat{\sigma}|^{2+2 \varepsilon}+2^{\varepsilon}\left|\sigma^{*}\right|^{2+2 \varepsilon} \int d \hat{\sigma} \exp \left(-|\hat{\sigma}|^{2}\right) \\
& \quad \leq \gamma_{1}(\varepsilon) t^{-1+\varepsilon}(8 \pi t)^{-p / 2} \exp \left(-\left|s^{\prime}-s^{\prime \prime}\right|^{2} 8 / t\right) \\
& \quad+\gamma_{2}(\varepsilon) t^{-1+\varepsilon}(8 \pi t)^{-p / 2}\left[\left|s^{\prime}+s^{\prime \prime}\right|^{2} \mid 8 t\right]^{1+\varepsilon} \exp \left(-\left|s^{\prime}-s^{\prime \prime}\right|^{2} / 8 t\right) .
\end{aligned}
$$


Here Hoelders inequality has been employed. Hence (2.22) can be estimated as follows:

$$
\begin{aligned}
& \iint d s d t\left|\int d s^{\prime}\right| \Omega_{1}\left(s-s^{\prime} ; t\right)|| s-\left.\left.s^{\prime}\right|^{1+\varepsilon} u\left(s^{\prime}\right)\right|^{2} \\
& +\gamma_{1}(\varepsilon) \int_{0}^{1} d t t^{\varepsilon-1} \iint d s^{\prime} d s^{\prime \prime} \overline{u\left(s^{\prime}\right)} u\left(s^{\prime \prime}\right)(8 \pi t)^{-p / 2} \exp \left(-\left|s^{\prime}-s^{\prime \prime}\right|^{2} / 8 t\right) \\
& +\gamma_{2}(\varepsilon) \int_{0}^{1} d t t^{\varepsilon-1} \iint d s^{\prime} d s^{\prime \prime} \overline{u\left(s^{\prime}\right)} u\left(s^{\prime \prime}\right) \\
& \quad \times(8 \pi t)^{-p / 2}\left(\left|s^{\prime}-s^{\prime \prime}\right|{ }^{2} / 8 t\right)^{1+\varepsilon} \exp \left(-\left|s^{\prime}-s^{\prime \prime}\right|{ }^{2} / 8 t\right) \\
& \leq \gamma(\varepsilon) \int_{0}^{1} d t t^{\varepsilon-1} \int|u|^{2} d s=\gamma(\varepsilon)^{\varepsilon-1} \int|u(s)|^{2} d s .
\end{aligned}
$$

Here again Lemma 2.1 and Lemma 1.1 were employed. A quite analogous argument is possible for $\Omega_{2}\left(s-s^{\prime} ; t\right)$; therefore Lemma 2.5 is proved.

LEMMA 2.6.

$$
\iint t^{2} d s d t\left|\int \Omega\left(s-s^{\prime} ; t\right) u\left(s^{\prime}\right) d s^{\prime}\right|^{2} \leq c \int|u(s)|^{2} d s
$$

for arbitrary $u(s)$ with compact carrier squared integrable over the whole $s$-space.

Proof. Again it suffices to prove this inequality for $\Omega$ replaced by $\Omega_{2}$ and $\Omega_{2}$. Now

$$
\begin{aligned}
& \int d s \Omega_{1}\left(s-s^{\prime} ; t\right) \Omega_{1}\left(s-s^{\prime \prime} ; t\right) \\
& =(2 t)^{-2}\left(8 \pi^{2} t\right)^{-p / 2} \exp \left(-\left|s^{\prime}-s^{\prime \prime}\right|^{2} / 8 t\right) \int d \hat{\sigma} \exp \left(-|\hat{\sigma}|^{2}\right) \\
& =(2 t)^{-2}(8 \pi t)^{p / 2} \exp \left(-\left|s^{\prime}-s^{\prime \prime}\right|^{2} / 8 t\right) .
\end{aligned}
$$

Hence by Lemma 2.1:

$$
\begin{aligned}
& \iint d s^{\prime} d s^{\prime \prime} \overline{u\left(s^{\prime}\right)} u\left(s^{\prime \prime}\right) \int d s \Omega_{1}\left(s-s^{\prime} ; t\right) \Omega_{1}\left(s-s^{\prime \prime} ; t\right) \\
& \quad \leq(2 t)^{-2} \int|u(s)|^{2} d s .
\end{aligned}
$$

Consequently

$$
\begin{aligned}
& \iint t^{2} d s d t\left|\int \Omega_{1}\left(s-s^{\prime} ; t\right) u\left(s^{\prime}\right) d s^{\prime}\right|^{2} \\
& \quad \leq 1 / 4 \int|u(s)|^{2} d s .
\end{aligned}
$$


Again a similar argument proves the corresponding inequality for $\Omega_{2}$; therefore Lemma 2.6 is proved.

We finally use the preceding lemmata to establish

\section{LEMMA 2.7. Let}

$$
A(s ; t)=\left(\left(a_{i k}(s ; t)\right)\right)
$$

be an $m \times m$-matrix with coefficients $a_{i k}(s ; t)$ having uniformly Hoelder continuous and uniformly bounded first partial derivatives in the domain

$$
D_{0}=\left\{s_{1}, \cdots, s_{p} ; t \ni-\infty<s_{k}<+\infty, k=1, \cdots, p ; 0<t<1\right\} .
$$

Let

$$
u(s)=\left(u_{1}(s), \cdots, u_{m}(s)\right)
$$

be an $m$-component vector function having a compact carrier and being squared integrable over the whole $\left(s_{1}, \cdots, s_{p}\right)$-space. Let the vector function

$$
A(s ; 0) u(s)=v(s)
$$

be Lipschitz continuous over the whole $\left(s_{1}, \cdots, s_{p}\right)$-space.

Statement. There exist two constants $c_{1}, c_{2}$ which are independent of $u(s)$ such that

$$
\begin{aligned}
& \iint d s d t\left|A(s ; t) \int d s^{\prime} \Omega\left(s-s^{\prime} ; t\right) u\left(s^{\prime}\right)\right|^{2} \\
& \quad \leq c_{1} \int|u(s)|^{2} d s+\left.c_{2} \sum_{i=1}^{p} \int|\partial v| \partial s_{i}\right|^{2} d s .
\end{aligned}
$$

Proof. We decompose as follows:

$$
\begin{aligned}
& A(s ; t) \int d s^{\prime} \Omega\left(s-s^{\prime} ; t\right) u\left(s^{\prime}\right)=\int \Omega\left(s-s^{\prime} ; t\right) v\left(s^{\prime}\right) d s^{\prime} \\
& \quad+(A(s ; t)-A(s ; 0)) \int \Omega\left(s-s^{\prime} ; t\right) u\left(s^{\prime}\right) d s^{\prime} \\
& \quad+\sum_{i=1}^{p} \int \Omega\left(s-s^{\prime} ; t\right)\left(s_{i}-s_{i}^{\prime}\right) u_{i}\left(s^{\prime}\right) d s^{\prime} \\
& \quad+\int \Omega\left(s-s^{\prime} ; t\right)\left[A(s ; 0)-A\left(s^{\prime} ; 0\right)\right. \\
& \left.\quad-\sum_{i=1}^{p}\left(s_{i}-s_{i}^{\prime}\right) \partial / \partial s_{i}^{\prime} A\left(s^{\prime} ; 0\right)\right] u\left(s^{\prime}\right) d s^{\prime}
\end{aligned}
$$

where 


$$
v(s)=A(s ; 0) u(s), u_{i}(s)=\left[\partial / \partial s_{i}(A(s ; 0))\right] u(s) .
$$

By our assumption for $A(s ; t)$ we get

$$
|(A(s ; t)-A(s ; 0)) w| \leq c t|w|
$$

and

$$
\begin{aligned}
& \left|\left[A(s ; 0)-A\left(s^{\prime} ; 0\right)-\sum_{i=1}^{p}\left(s_{i}-s_{i}^{\prime}\right) \partial / \partial s_{i}\left(A\left(s^{\prime} ; 0\right)\right)\right] u\left(s^{\prime}\right)\right| \\
& \quad \leq c\left|s-s^{\prime}\right|^{1+\varepsilon}\left|u\left(s^{\prime}\right)\right| .
\end{aligned}
$$

Therefore we can use the Lemmata $2.3,2.4,2.5$, and 2.6 respectively to estimate the integrals in (2.33) for the succeeding terms in (2.34) by either $c \int|u(s)|^{2} d s$ or $\left.\int|\partial v| \partial s_{i}\right|^{2} d s$. Hence Lemma 2.7 is proved.

LEMMA 2.8. Let $u(s)$ be a bounded measurable m-component vector function defined in the whole s-space and let it have a compact carrier. Further, with the notations of Lemma 2.7, let

$$
v(s)=A(s ; 0) u(s)
$$

be Lipschitz continuous over the whole s-space.

Let

$$
u(s ; t)=\int \Phi\left(s-s^{\prime} ; t\right) u\left(s^{\prime}\right) d s^{\prime}
$$

Then

$$
\lim _{t \rightarrow 0} u(s ; t)=u(s) \text { almost everywhere }
$$

and

$$
v(s ; t)=A(s ; t) u(s ; t)
$$

is continuous all over in the domain $D_{0}$ defined in (2.30) and its boundary.

Proof. Let $\varepsilon>0$ be given. Since $u(s)$ is bounded and measurable, by Lusin's theorem a measurable set $E_{\varepsilon}$ of $p$-dimensional measure $m\left(E_{\varepsilon}\right)$ less than $\varepsilon$ exists such that $u(s)$ is continuous on the complement $C\left(E_{\varepsilon}\right)$ of $E_{\text {s }}$ with respect to the $s$-space. If $\chi(s)$ denotes the characteristic function of $E_{\varepsilon}$ and if $\Delta$ denotes the cube with sides $2 \delta$ defined in (1.8), then by well known facts

$$
\lim _{\delta \rightarrow 0} \delta^{-p} \int_{\Delta} \chi(s) d s=0
$$

for every $s_{0} \in C\left(E_{\varepsilon}+N_{\varepsilon}\right)$ where $N_{\varepsilon}$ denotes a certain nullset. We will show that for every $s_{0} \in C\left(E_{\varepsilon}+E_{\varepsilon}\right)$ relation (2.40) holds. This will 
prove the first statement of the lemma, because then obviously it is possible to construct a monotonically decreasing sequence of sets which converges toward a nullset and such that after exempting any set of the sequence the statement $(2.40)$ holds.

Now, $s_{0} \in C\left(N_{\varepsilon}+E_{\varepsilon}\right)$ being given, decompose as follows:

$$
\int \Phi\left(s_{0}-s^{\prime} ; t\right) u\left(s^{\prime}\right) d s^{\prime}=\int_{C\left(E_{\varepsilon}\right) \cap \Delta_{0}}+\int_{E_{\varepsilon} \cap \Delta_{0}}+\int_{O\left(s_{0}\right)}
$$

where $\Delta_{0}$ denotes the cube (1.8) with side $\delta=\delta_{0}$. Then

$$
\int_{\sigma\left(E_{\varepsilon}\right) \cap s_{0}}=\mu_{\Delta_{0}} \int_{\sigma\left(E_{\varepsilon}\right) \cap \Delta_{0}} \Phi\left(s_{0}-s^{\prime} ; t\right) d s^{\prime}
$$

where $\mu_{\Delta_{0}}$ denotes a mean value of $u(s)$ in the cube $\Delta_{0}$.

But since $u$ is continuous in $C\left(E_{\varepsilon}\right) \cap \Delta$ it follows that

$$
\left|\mu_{\Delta_{0}}-u\left(s_{0}\right)\right|<\varepsilon^{\prime}
$$

if $\delta_{0}>0$ is sufficiently small. Also

$$
\int_{C\left(E_{\varepsilon}\right) \cap s_{0}} \Phi\left(s_{0}-s^{\prime} ; t\right) d s^{\prime} \leq \int \Phi\left(s_{0}-s^{\prime} ; t\right) d s^{\prime}=1 .
$$

Consequently, using (2.44) and (2.46) we get

$$
\begin{aligned}
& \left|\int_{C\left(E_{\varepsilon}\right) \cap \Delta_{0}} \Phi\left(s_{0}-s^{\prime} ; t\right) u\left(s^{\prime}\right) d s^{\prime}-u\left(s_{0}\right)\right| \\
& \quad \leq\left|\mu_{\Delta_{0}}-u\left(s_{0}\right)\right|+c \int_{\Delta_{0}} \Phi\left(s_{0}-s^{\prime} ; t\right) \chi\left(s^{\prime}\right) d s^{\prime} \\
& \quad+c \int_{C\left(\Delta_{0}\right)} \Phi\left(s-s^{\prime} ; t\right) d s^{\prime}
\end{aligned}
$$

with $c=\sup |u(s)|$. Finally for the second and third integral in (2.43) we obtain estimates

$$
\left|\int_{E_{\varepsilon} \cap \Delta_{0}}\right| \leq c \int_{\Delta_{0}} \Phi\left(s-s^{\prime} ; t\right) \chi\left(s^{\prime}\right) d s^{\prime}
$$

and

$$
\left|\int_{C\left(\Delta_{0}\right)}\right| \leq c \int_{C\left(\Delta_{0}\right)} \Phi\left(s-s^{\prime} ; t\right) d s^{\prime} .
$$

Hence by (2.43), (2.47), (2.48), and (2.49)

$$
\begin{aligned}
& \left|\int \Phi\left(s-s^{\prime} ; t\right) u\left(s^{\prime}\right) d s^{\prime}-u\left(s_{0}\right)\right| \\
& \leq\left|\mu_{\Delta_{0}}-u\left(s_{0}\right)\right| 2 c \int_{\Delta_{0}} \Phi\left(s-s^{\prime} ; t\right) \chi\left(s^{\prime}\right) d s^{\prime} \\
& \quad+2 c \int_{\sigma\left(\Delta_{0}\right)} \Phi\left(s-s^{\prime} ; t\right) d s^{\prime} .
\end{aligned}
$$


Choosing first $\delta_{0}$ sufficiently small the first term can be made arbitrarily small; then keeping $\delta_{0}$ fixed by Lemma 1.2 and (2.42) the second term also can be made arbitraily small by choosing $t$ small. Also the last term for fixed $\delta_{0}$ becomes arbitrarily small if $t$ tends to zero. Hence formula (2.40) is proved.

In order to prove the continuity of (2.41) we decompose

$$
\begin{aligned}
v(s ; t) & =\int \Phi\left(s-s^{\prime} ; t\right) v\left(s^{\prime}\right) d s^{\prime} \\
& +\int \Phi\left(s-s^{\prime} ; t\right)\left(A(s ; t)-A\left(s^{\prime} ; 0\right)\right) u\left(s^{\prime}\right) d s^{\prime} .
\end{aligned}
$$

Since $v(s)$ is assumed to be Lipschitz continuous, the first term obuiously is a continuous function in $D_{0}$. The second term is also continuous for every $t>0$. But since $u(s)$ is assumed to be bounded we get

$$
\begin{aligned}
& \int \Phi\left(s-s^{\prime} ; t\right)\left(A(s ; t)-A\left(s^{\prime} ; 0\right)\right) u\left(s^{\prime}\right) d s^{\prime} \\
\leq & c t \int \Phi\left(s-s^{\prime} ; t\right) d s^{\prime}+c^{\prime} \int \Phi\left(s-s^{\prime} ; t\right)\left|s-s^{\prime}\right| d s^{\prime} \\
= & c^{\prime \prime} t+c^{\prime} \mid t^{1 / 2} \longrightarrow
\end{aligned}
$$

Therefore the continuity is also proved for $t=0$. This proves the lemma.

3. A continuation theorem. Let $D$ be a bounded domain of the $\left(x_{1}, \cdots, x_{n}\right)$-space with a twice continuously differentiable boundary $\Gamma$ which consits of a finite number of simple nonintersecting hyper surfaces. More specifically we assume that the boundary $\Gamma$ has second derivatives satisfying a uniform Hoelder condition. Let

$$
a_{i}(x)=\left(\left(a_{j l}^{i}(x)\right)\right), i=1, \cdots, n, b(x)=\left(\left(b_{i k}(x)\right)\right)
$$

be $m \times m$-matrices with complex coefficients defined in $D+\Gamma$. Let $a_{i}(x)$ be hermitian symmetric and its coefficients be continuously differentiable in $D+\Gamma$ and let the derivatives satisfy a uniform Hoelder condition in $D+I$. Let $b(x)$ have continuous coefficients in $D+\Gamma$. Let $A(x), x \in D+\Gamma$ be any hermitian symmetric $m \times m$-matrix having continuously differentiable coefficients in $D+\Gamma$ and such that

$$
A(x)=\sum_{i=1}^{n} a_{i}(x) \nu_{i}(x), x \in \Gamma
$$

where $\nu(x)=\left(\nu_{1}(x), \cdots, \nu_{n}(x)\right)$ denotes the exterior normal on $\Gamma$. We define the differential operator $L_{1}$ in $\mathfrak{D}_{L_{1}}$ by

$$
L_{1} u=\sum_{i=1}^{n} a_{i}(x) \partial u / \partial x_{i}+b(x) u(x)
$$


for complex valued $m$-component vector functions

$$
u(x)=\left(u_{1}(x), \cdots, u_{n}(x)\right)
$$

where $\mathfrak{D}_{L_{1}}$ is the space of all $u(x)$ satisfying the following conditions:

(a) $u, \partial u / \partial x_{i}, i=1, \cdots, n$, continuous in $D$.

(b) $u(x)$ uniformly bounded in $D$.

(c) $\lim _{\varepsilon \rightarrow 0} u(x-\varepsilon \nu)=u(x)$ for every $x \in \Gamma$, except possibily on an $n$-1-dimensional null set.

(d) $v(x)=A(x) u(x)$ is continuous on $D+\Gamma$

(e) $\int_{D}\left|L_{1} u\right|^{2} d x<\infty$.

We prove the following

THEOREM 3.1. Let $u_{0}(x)$ be an m-component vector function which is defined measurable and bounded on $\Gamma$ and for which

$$
v_{0}(x)=A(x) u_{0}(x)
$$

is Lipschitz continuous on $\Gamma$.

Then there exists a function $u(x) \in \mathfrak{D}_{L_{1}}$ such that

$$
u(x)=u_{0}(x) \text { on } \Gamma \text {. }
$$

Proof. We consider any arbitray point $x_{0} \in \Gamma$. There is a certain neighborhood

$$
U_{x_{0}}=\left\{x \ni\left|x-x_{0}\right| \leq \varepsilon\right\}
$$

which can be mapped by a twice Hoelder continuously differentiable one to one mapping

$$
y=y(x)
$$

onto a bounded region in the $\left(y_{1}, \cdots, y_{n}\right)$-space in such a way that the point $x_{0}$ goes into the origin $y=(0, \cdots, 0)$, the intersection

$$
\Gamma_{x}=\Gamma_{0} \cap U_{x_{0}}
$$

into a certain neighborhood of $(0, \cdots, 0)$ on the hyperplane $y_{1}=0$, and the intersection

$$
D_{x_{0}}=(D+\Gamma) \cap U_{x_{0}}
$$

into a certain half neighborhood of $(0, \cdots, 0)$ satisfying $y_{1} \geq 0$. We also can assume that the Jacobian does not vanish.

$$
\operatorname{det}\left(\left(\partial y_{i} / \partial x_{k}\right)\right) \neq 0, y \in D_{x_{0}}+\Gamma_{x_{0}} .
$$


The image $y\left(D_{x_{0}}\right)$ of $D_{x_{0}}$ under this transformation contains a cube of the type

$$
\mathfrak{Q}_{x_{0}}=\left\{y \in 0 \leq y_{1} \leq \eta\left(x_{0}\right),\left|y_{\nu}\right| \leq 1 / 2 \eta\left(x_{0}\right), \nu=2, \cdots, n\right\} .
$$

We denote the intersection of $\mathfrak{l}_{x_{0}}$ with the hyperplane $y_{1}=0$ by $\mathfrak{q}_{x_{0}}$ and we set

$$
x\left(\mathfrak{Q}_{x_{0}}\right)=\mathfrak{Q}_{x_{0}}^{\prime}, x\left(\mathfrak{q}_{x_{0}}\right)=\mathfrak{q}_{x_{0}}^{\prime}
$$

where $x=x(y)$ denotes the inverse transformation of (3.8). There is a hypersphere

$$
U_{x_{0}}^{\prime}=\left\{x \ni\left|x-x_{0}\right| \leq \eta^{\prime}\left(x_{0}\right)\right\}
$$

such that

$$
D_{x_{0}}^{\prime}=D_{x_{0}} \cap U_{x_{0}}^{\prime} \subset \mathfrak{D}_{x_{0}}^{\prime}
$$

and such that the same inclusion still holds for $\eta^{\prime}\left(x_{0}\right)$ being replaced by a somewhat larger number.

This construction can be employed for every $x_{0} \in I$. Since $\Gamma$ is a bounded closed set, the whole $\Gamma$ can be covered by the interior points of a finite number of spheres

$$
U_{x_{\nu}}^{\prime}, \nu=1, \cdots, N \text {. }
$$

There is a decomposition of the identity, i. e., a set of $N$ functions

$$
\varphi_{\nu}(x), \nu=1, \cdots, N
$$

being defined and infinitely differentiable in the whole $\left(x_{1}, \cdots, x_{n}\right)$-space and such that

$$
\varphi_{\nu}(x)=0 \text { outside of } U_{x_{\nu}}^{\prime}
$$

and

$$
\sum_{\nu=1}^{N} \varphi_{\nu}(x)=1 \text { on } \Gamma
$$

Now any vector function $u_{0}(x)$ being given which satisfies the conditions of the Theorem 3.1, define

$$
u_{\nu, 0}(x)=u_{0}(x) \varphi_{\nu}(x), x \in \Gamma, \nu=1, \cdots, N .
$$

Clearly $u_{\nu, 0}(x)$ also satisfies the assumptions of Theorem 3.1, especially because

$$
A(x) u_{\nu, 0}(x)=\left(A(x) u_{0}(x)\right) \varphi_{\nu}(x) .
$$

We will prove that every $u_{\nu, 0}(x)$ can be continued to a function $u_{\nu}(x) \in \mathscr{D}_{L_{1}}$ 
in the sense of the assertion. This obviously will prove Theorem 3.1, because the sum of all $u_{\nu}(x)$ will be the desired continuation of $u_{0}(x)$.

Now, if we apply the mapping just defined in each particular neighborhood $D_{x_{\nu}}$ then the vector function $u_{\nu, 0}(x)$ will be transformed into a certain function

$$
w_{\nu, 0}(y)=u_{\nu, 0}(x(y))
$$

defined and measurable on $y\left(\Gamma_{x_{\nu}}\right)$. which contains the cube $\mathfrak{q}_{x_{\nu}}$. Since by definition $u_{\nu, 0}(x)=0$ outside of $D_{x_{\nu}}^{\prime}$ and since

$$
y\left(D_{x_{\nu}}^{\prime}\right) \subset \mathfrak{\Omega}_{x_{\nu}}
$$

holds, the function $w_{\nu, 0}(y)$ is defined for $y \in \mathfrak{q}_{x_{\nu}}$ and has its carrier in the interior of this $n$-1-dimensional cube. We can consider $w_{\nu, 0}(y)$ as being defined on the whole hyperplane $y_{1}=0$ by setting it equal to zero outside of $q_{x_{\nu}}$. We would like to apply the various lemmata of $\S 2$. In order to do this we first transform the operator $L_{1}$ to the new variables $y$.

$$
L_{1}=\sum_{i=1}^{n} \tilde{a}_{i}(y) \partial / \partial y_{i}+\tilde{b}(y), y \in y\left(D_{x_{\nu}}\right)
$$

where

$$
\tilde{a}_{i}(y)=\sum_{k=1}^{n} \partial y_{i} / \partial x_{k} a_{k}(x(y)) ; \tilde{b(y)}=b(x(y)) .
$$

Further we define

$$
\tilde{A}(y)=A(x(y)), y \in y\left(D_{x_{\nu}}\right),
$$

Clearly it is possible to continue the matrix $\tilde{A}(y)$ to a matrix function being defined, bounded and continuously differentiable on the whole semispace

$$
y_{1} \geq 0,-\infty<y_{\nu}<+\infty, \nu=2, \cdots, n ;
$$

its first derivatives satisfying a uniform Hoelder condition in every compact subregion. Now we remark that for

$$
y_{1}=t, y_{2}=s_{1}, y_{3}=s_{2}, \cdots, y_{n}=s_{p} ; p=n-1
$$

the functions $w_{\nu, 0}(y)$ and $\tilde{A}(y)$ satisfy every assumption necessary for application of Lemma 2.2, Lemma 2.7, and Lemma 2.8. Hence the function

$$
w_{\nu}(y)=\int \Phi\left(s-s^{\prime} ; y_{1}\right) w_{\nu, 0}\left(s^{\prime}\right) d s^{\prime}
$$

satisfies the following conditions: 
$(\alpha) w_{\nu}, \partial w_{\nu} / \partial y_{i}$ continuous for $y_{1}>0$.

( $\beta) \quad w_{\nu}$ uniformly bounded for $y_{1} \geq 0$.

( $\gamma) \lim _{\Sigma \rightarrow 0} w_{\nu}(y-\varepsilon z)$ exists for every $y$ with $y_{1}=0$ and every vector $z_{1}=1, z_{j}=0, j=2, \cdots, n$ with the possible exemption of a set of $n$-1-dimensional measure zero which is contained in $\mathfrak{q}_{x_{\nu}}$.

( $\delta) v_{\nu}(y)=\tilde{A}(y) w_{\nu}(y)$ is continuous for $y_{1} \geq 0$.

$(\varepsilon)$

$$
\int_{y_{1} \geq 0}\left\{\left|w_{\nu}(y)\right|^{2}+\left|\tilde{A(y)} \partial w_{\nu} / \partial y_{1}\right|^{2}+\sum^{n}\left|\partial w_{\nu} / \partial y_{i}\right|^{2}\right\} d y<\infty
$$

Finally take any infinitely differentiable function $\tilde{\varphi}_{\nu}(y)$ being $=1$ on $y\left(D_{x_{\nu}}^{\prime}\right)$ and having its carrier in $y\left(D_{x_{\nu}}\right)$ and take

$$
\tilde{w}_{\nu}(y)=\tilde{\varphi}_{\nu}(y) w(y) .
$$

Clearly $\tilde{w}_{\nu}(y)$ also has the properties $(\alpha), \cdots,(\varepsilon)$. Transform this function back to the old variables and continue it zero outside of $D_{x_{\nu}}(x)$. Call the new function $u_{\nu}(x)$. Then it is clear that

$$
u_{\nu}(x)=u_{\nu, 0}(x) \text { on } \Gamma \text {. }
$$

Also $u_{2}(x)$ satisfies the conditions $(a),(b),(c)$, and $(d)$. Since

$$
\left|L_{1} u_{\nu}\right|^{2} \leq c\left[\left|\tilde{A}(y) \partial u_{\nu} / \partial y_{1}\right|^{2}+\sum_{i=2}^{n}\left|\partial u_{\nu} / \partial y_{i}\right|^{2}+\left|u_{\nu}\right|^{2}\right]
$$

(3.30) yields the condition $(e)$ too. Hence $u_{\nu}(x)$ is the desired continuation and Theorem 3.1 is proved.

4. A counterexample. Let $D$ be the unit circle $x_{1}^{2}+x_{2}^{2}<1$ and accordingly $\Gamma$ be the periphery of the unit circle $x_{1}^{2}+x_{2}^{2}=1$. In $D$ we consider the operator defined in formula (5) of the introduction

$$
L_{1}=a_{1} \partial / \partial x_{1}+a_{2} \partial / \partial x_{2}
$$

with

$$
a_{1}=\left(\begin{array}{rr}
-1 & 0 \\
0 & 1
\end{array}\right), \quad a_{2}=\left(\begin{array}{ll}
0 & 1 \\
1 & 0
\end{array}\right)
$$

Then the equation

$$
L_{1} u=f
$$

for the 2-component vector functions

$$
u=\left\{u_{1}, u_{2}\right\}, f=\left\{f_{1}, f_{2}\right\}
$$

defined in $D+\Gamma^{\prime}$ is equivalent to the system 


$$
\begin{aligned}
-\partial u_{1} / \partial x_{1}+\partial u_{2} / \partial x_{2} & =f_{1} \\
\partial u_{1} / \partial x_{2}+\partial u_{2} / \partial x_{1} & =f_{2} .
\end{aligned}
$$

Hence for real valued $u_{1}, u_{2}$ we get

$$
\begin{aligned}
& \int_{D}\left(f_{1}^{2}+f_{2}^{2}\right) d x=\int_{D}\left(\partial u_{1} / \partial x_{1}-\partial u_{2} / \partial x_{2}\right)^{2}+\left(\partial u_{1} / \partial x_{2}+\partial u_{2} / \partial x_{1}\right)^{2} d x \\
& =\int_{D}\left[\left(\partial u_{1} / \partial x_{1}\right)^{2}+\left(\partial u_{1} / \partial x_{2}\right)^{2}+\left(\partial u_{2} / \partial x_{1}\right)^{2}+\left(\partial u_{2} / \partial x_{2}\right)^{2}\right] d x \\
& \quad+2 \int_{D}\left(\partial u_{1} / \partial x_{2} \partial u_{2} / \partial x_{1}-\partial u_{1} / \partial x_{1} \partial u_{2} / \partial x_{2}\right) d x
\end{aligned}
$$

Now, assuming $u$ twice continuously differentiable in $D+\Gamma$ we can apply Green's formula to the last integral:

$$
\begin{aligned}
& \int_{D}\left(\partial u_{1} / \partial x_{2} \partial u_{2} / \partial x_{1}-\partial u_{1} / \partial x_{1} \partial u_{2} / \partial x_{2}\right) d x \\
= & \int_{\Gamma} u_{1}\left(x_{2} \partial u_{2} / \partial x_{1}-x_{1} \partial u_{2} / \partial u_{2} / \partial x_{2}\right) d \sigma .
\end{aligned}
$$

Hence the last integral in (4.6) is equal to

$$
2 \int_{\Gamma} u_{1}\left(x_{2} \partial u_{2} / \partial x_{1}-x_{1} \partial u_{2} / \partial x_{2}\right) d \sigma=-2 \int_{0}^{2 \pi} u_{1} \partial u_{2} / \partial \vartheta d \vartheta
$$

where

$$
\vartheta=\operatorname{arctg} x_{2} / x_{1} .
$$

Now we impose on $u$ the condition

$$
u_{1} \sin \vartheta / 2+u_{2} \cos \vartheta / 2=0 \text {. }
$$

Then

$$
\begin{aligned}
& -2 \int_{0}^{2 \pi} u_{1} \partial u_{2} / \partial \vartheta d \vartheta=\int_{0}^{2 \pi}\left[\partial / \partial \vartheta\left(u_{2}^{2}\right)\right] \cot \vartheta / 2 \\
= & -\int_{0}^{2 \pi} u_{2}^{2} \partial / \partial \vartheta(\cot \vartheta / 2) d \vartheta=1 / 2 \int_{0}^{2 \pi} u_{2}^{2} \sin ^{-2} \vartheta / 2 d \vartheta .
\end{aligned}
$$

This integration by parts is legitimate because the condition (4.10) implies $u_{2}=0$ at $\vartheta=0,2 \pi$. Since $u$ is supposed to have continuous first derivatives it follows that $u_{2}^{2} \sin ^{-2} \vartheta / 2$ remains bounded also for $\vartheta=0,2 \pi$. Consequently

$$
\begin{aligned}
& \int_{D}\left|L_{1} u\right|^{2} d x=\int_{D}|f|^{2} d x \\
= & \int_{D}\left[\left(\partial u_{1} / \partial x_{1}\right)^{2}+\left(\partial u_{1} / \partial x_{2}\right)^{2}+\left(\partial u_{2} / \partial x_{1}\right)^{2}+\left(\partial u_{2} / \partial x_{2}\right)^{2}\right] d x \\
& +1 / 2 \int_{0}^{2 \pi} u_{2}^{2} \sin ^{-2} \vartheta / 2 d \vartheta .
\end{aligned}
$$

Since the last integral is nonnegative we obtain 


$$
\begin{aligned}
\int_{D}\left|L_{1} u\right|^{2} d x \geq & \int_{D}\left[\left(\partial u_{1} / \partial x_{1}\right)^{2}+\left(\partial u_{1} / \partial x_{2}\right)^{2}\right. \\
& \left.+\left(\partial u_{2} / \partial x_{1}\right)^{2}+\left(\partial u_{2} / \partial x_{2}\right)^{2}\right] d x .
\end{aligned}
$$

Next assume $\phi=\left\{\varphi_{1}, \varphi_{2}\right\}$ to be some function satisfying the conditions (a), (b), (c), and (e), of Theorem 3.1 applied to the special operator $L_{1}$ defined in (4.1). Also assume that on the boundary $\Gamma$ :

$$
\varphi_{1}=\alpha(\vartheta) \cos \vartheta / 2, \varphi_{2}=-\alpha(\vartheta) \sin \vartheta / 2,0 \leq \vartheta \leq 2 \pi .
$$

Let $\alpha(\vartheta)$ be real valued and piecewise continuous but not continuous. Then we will show that this leads to a contradiction.

First of all the vector function $\varphi$ can be assumed to be real valued in $D+\Gamma$ because any complex valued such $\varphi$ being given, $1 / 2(\varphi+\bar{\varphi})$ would satisfy the same conditions as $\varphi$ and would be real valued.

Now, if $L_{-}$in $\mathfrak{D}_{L_{-}}$denotes the restriction of the operator $L_{1}$ in $\mathfrak{D}_{L_{1}}$ to the space $\mathfrak{D}_{L_{-}}$of all functions twice continuously differentiable in $D+\Gamma$ and satisfying the boundary conditions (4.10) then we obtain a dissipative operator in the sense of R. S. Phillips [4], which is characterized by local boundary conditions. For the matrix

$$
A=\sum_{i=1}^{2} a_{i} \nu_{i}=a_{1} \cos \vartheta+a_{2} \sin \vartheta
$$

we get the representation

$$
\begin{aligned}
A(\vartheta)= & \left(\begin{array}{r}
-\cos \vartheta \sin \vartheta \\
\sin \vartheta \cos \vartheta
\end{array}\right)=\left(\begin{array}{l}
\sin ^{2} \vartheta / 2, \sin \vartheta / 2 \cos \vartheta / 2 \\
\sin \vartheta / 2 \cos \vartheta / 2, \cos ^{2} \vartheta / 2
\end{array}\right) \\
& -\left(\begin{array}{c}
\cos ^{2} \vartheta / 2,-\sin \vartheta / 2 \cos \vartheta / 2 \\
-\sin \vartheta / 2 \cos \vartheta / 2, \sin ^{2} \vartheta / 2
\end{array}\right)
\end{aligned}
$$

and it is easy to see that the two matrices of this last decomposition are identical with the matrices $P_{0}$ and $N_{0}$ respectively which project orthogonally onto the spaces of all eigenvectors corresponding to the eigenvalues +1 and -1 respectively. The boundary condition

$$
P_{0} u=0 \text { on } \Gamma
$$

obviously is equivalent to the condition (4.10). Hence the inner product $\bar{u} A u$ is $\leq 0$ for all $u$ satisfying the condition (4.17) (or (4.10)). Hence

$$
Q(u, u)=2 \operatorname{Re} \int_{D} \bar{u} L_{1} u d x=\int_{\Gamma} \bar{u} A u d \sigma \leq 0,
$$

which proves that $L_{-}$in $\mathfrak{D}_{L_{-}}$is dissipative. On the other hand in the sense of K. O. Kriedrichs [3] this boundary condition is "admissible", because 


$$
A=P_{0}-N_{0}, P_{0} \geq 0, N_{0} \geq 0 .
$$

Also the rank of $A$ is constantly equal to two.

Hence if $L_{-}^{*}$ in $\mathfrak{D}_{L_{-}^{*}}$ denotes the adjoint of $L_{-}$in $\mathfrak{D}_{L_{-}}$with respect to the inner product

$$
\langle u, v\rangle=\int_{D} \bar{u} v d x
$$

and if $L_{+}$in $\mathfrak{D}_{L_{+}}$denotes the operator analogous to $L_{-}$in $\mathfrak{D}_{L_{-}}$with the boundary condition (4.17) replaced by $N_{0} u=0, x \in \Gamma$, then

$$
L_{-}^{* *}=L_{+}^{*} \text {. }
$$

But $\varphi$ is a function of $L_{+}^{*}$ because from the conditions (a), (b), (c) and (e) it follows immediately that

$$
\langle\varphi, L u\rangle+\langle L \varphi, u\rangle=\int_{\Gamma} \bar{\varphi} A u d \sigma=0
$$

for all $u \in \mathfrak{D}_{L_{+}}$. Hence (4.21) implies

$$
\varphi \in \mathfrak{D}_{L_{-}^{* *}}
$$

Therefore a sequence $\varphi^{n} \in \mathfrak{D}_{L_{-}}$exists such that

$$
\begin{gathered}
\left\langle\varphi^{n}-\varphi, \varphi^{n}-\varphi\right\rangle \longrightarrow 0, n \longrightarrow \infty \\
\left\langle L_{1}\left(\varphi^{n}-\varphi\right), L_{1}\left(\varphi^{n}-\varphi\right)\right\rangle \longrightarrow 0, n \longrightarrow \infty .
\end{gathered}
$$

Now (4.25) implies

$$
\left\langle L_{1}\left(\varphi^{n}-\varphi^{m}\right), L_{1}\left(\varphi^{n}-\phi^{m}\right)\right\rangle \longrightarrow 0, n, m \longrightarrow \infty .
$$

Let

$$
\varphi^{n, m}=\varphi^{n}-\phi^{m}
$$

then (4.13) yields

$$
\left\langle\partial \varphi^{n m} / \partial x_{1}, \partial \varphi^{n m} / \partial x_{1}\right\rangle+\left\langle\partial \varphi^{n m} / \partial x_{2}, \partial \varphi^{n m} / \partial x_{2}\right\rangle \longrightarrow 0, n, \mathrm{~m} \rightarrow \infty .
$$

Hence $\partial \varphi^{n} / \partial x_{1}, \partial \varphi^{n} / \partial x_{2}$ converges in the square mean. Let

$$
\partial \varphi^{n} / \partial x_{1} \longrightarrow \psi, n \longrightarrow \infty,
$$

and let $u$ be any vector function continuously differentiable in $D+\Gamma$ and vanishing outside of some circle $|x| \leq r<1$. Then

$$
\left\langle\partial \varphi^{n} / \partial x_{1}, u\right\rangle=-\left\langle\varphi^{n}, \partial u / \partial x_{1}\right\rangle \text {. }
$$

For $n \rightarrow \infty$ we get 


$$
\langle\varphi, u\rangle=-\left\langle\mathcal{p}, \partial u / \partial x_{1}\right\rangle \text {. }
$$

But $\rho$ is continuously differentiable for $|x|<1$. Hence, using the special properties of $u$, we get

$$
\langle\psi, u\rangle=-\left\langle\varphi, \partial u \mid \partial x_{1}\right\rangle=\left\langle\partial \varphi / \partial x_{1}, u\right\rangle .
$$

Or

$$
\left\langle\psi-\partial \varphi \mid \partial x_{1}, u\right\rangle=0
$$

for all $u$ with the above properties. But the set of all such $u$ is dense in the space $L_{2}$; hence

$$
\psi=\partial \varphi / \partial x_{1} .
$$

In the same manner we obtain the relation

$$
\partial \varphi^{n} / \partial x_{2} \longrightarrow \partial \varphi / \partial x_{2}
$$

Hence the derivatives $\partial \varphi / \partial x_{1}, \partial \varphi / \partial x_{2}$ are squared integrable and the Dirichlet-integral of $\varphi$ exists.

But it is a well known fact that a function $\phi$ with the properties (a), (b), (c) which is piecewise continuous on the periphery of the unit circle and has a jump, cannot have the Dirichlet integral existing.

\section{REFERENCES}

1. H. O. Cordes, On maximal first order partial differential operators; to appear.

2. K. O. Friedrichs, The identity of weak and strong solutions of differential operators, Trans. Amer. Math. Soc., 55 (1944), 132-151.

3. K. O. Friedrichs, Symmetric positive linear differential equations, Dissipative Domm. Pure Appl. Nath., 11, (1958), 333-418.

4. R. R. Phillips, Dissipative operators and hyperbolic systems of partial differential operators; to appear in Pacific. J. Math,

5. I. Schur, Bemerkungen zur Theorie der beschrankten Billinearformen mit unendlich vielen Veranderlichen; J.f.d. reine u. angew. Math., 140, (1911), 1-28; especially page 6.

UNIVERSITY OF CALIFORNIA

Berkeley, CaLifornia 



\section{PACIFIC JOURNAL OF MATHEMATICS}

EDITORS

David Gilbarg

Stanford University

Stanford, California

R. A. Beaumont

University of Washington

Seattle 5 , Washington
A. L. Whiteman

University of Southern California

Los Angeles 7, California

L. J. PAIGE

University of California

Los Angeles 24, California

\section{ASSOCIATE EDITORS}

\author{
E. F. BECKENBACH \\ C. E. BURGESS \\ E. HEWITT \\ A. HORN
}

\author{
V. GANAPATHY IYER \\ R. D. JAMES \\ M. S. KNEBELMAN \\ L. NACHBIN
}
I. NIVEN
E. G. STRAUS
T. G. OSTROM
G. SZEKERES
H. L. ROYDEN
F. WOLF
M. M. SCHIFFER
K. YOSIDA

\section{SUPPORTING INSTITUTIONS}

\author{
UNIVERSITY OF BRITISH COLUMBIA \\ CALIFORNIA INSTITUTE OF TECHNOLOGY \\ UNIVERSITY OF CALIFORNIA \\ MONTANA STATE UNIVERSITY \\ UNIVERSITY OF NEVADA \\ OREGON STATE COLLEGE \\ UNIVERSITY OF OREGON \\ OSAKA UNIVERSITY \\ UNIVERSITY OF SOUTHERN CALIFORNIA
}

\author{
STANFORD UNIVERSITY \\ UNIVERSITY OF TOKYO \\ UNIVERSITY OF UTAH \\ WASHINGTON STATE COLLEGE \\ UNIVERSITY OF WASHINGTON \\ $* \quad * \quad *$ \\ AMERICAN MATHEMATICAL SOCIETY \\ CALIFORNIA RESEARCH CORPORATION \\ HUGHES AIRCRAFT COMPANY \\ SPACE TECHNOLOGY LABORATORIES
}

Mathematical papers intended for publication in the Pacific Journal of Mathematics should be typewritten (double spaced), and the author should keep a complete copy. Manuscripts may be sent to any one of the four editors. All other communications to the editors should be addressed to the managing editor, L. J. Paige at the University of California, Los Angeles 24, California.

50 reprints per author of each article are furnished free of charge; additional copies may be obtained at cost in multiples of 50 .

The Pacific Journal of Mathematics is published quarterly, in March, June, September, and December. The price per volume (4 numbers) is $\$ 12.00$; single issues, $\$ 3.50$. Back numbers are available. Special price to individual faculty members of supporting institutions and to individual members of the American Mathematical Society: $\$ 4.00$ per volume; single issues, $\$ 1.25$.

Subscriptions, orders for back numbers, and changes of address should be sent to Pacific Journal of Mathematics, 2120 Oxford Street, Berkeley 4, California.

Printed at Kokusai Bunken Insatsusha (International Academic Printing Co., Ltd.), No. 6, 2-chome, Fujimi-cho, Chiyoda-ku, Tokyo, Japan.

PUBLISHED BY PACIFIC JOURNAL OF MATHEMATICS, A NON-PROFIT CORPORATION

The Supporting Institutions listed above contribute to the cost of publication of this Journal, but they are not owners or publishers and have no responsibility for its content or policies. 


\section{Pacific Journal of Mathematics}

\section{Vol. 9, No. 4 \\ August, 1959}

Frank Herbert Brownell, III, A note on Kato's uniqueness criterion for

Schrödinger operator self-adjoint extensions ............... 953

Edmond Darrell Cashwell and C. J. Everett, The ring of number-theoretic

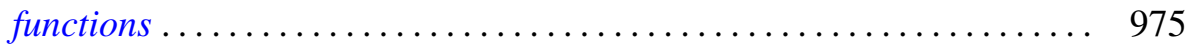

Heinz Otto Cordes, On continuation of boundary values for partial

differential operators ............................. 987

Philip C. Curtis, Jr., n-parameter families and best approximation . . . . . . 1013

Uri Fixman, Problems in spectral operators . . . . . . . . . . . . . . . 1029

I. S. Gál, Uniformizable spaces with a unique structure .............. 1053

John Mitchell Gary, Higher dimensional cyclic elements ............ 1061

Richard P. Gosselin, On Diophantine approximation and trigonometric

polynomials ..................................... 1071

Gilbert Helmberg, Generating sets of elements in compact groups ........ 1083

Daniel R. Hughes and John Griggs Thompson, The H-problem and the

structure of $H$-groups .................................. 1097

James Patrick Jans, Projective injective modules ................. 1103

Samuel Karlin and James L. McGregor, Coincidence properties of birth and

death processes ..................................... 1109

Samuel Karlin and James L. McGregor, Coincidence probabilities ........ 1141

J. L. Kelley, Measures on Boolean algebras ................... 1165

John G. Kemeny, Generalized random variables ................... 1179

Donald G. Malm, Concerning the cohomology ring of a sphere bundle ... . . 1191

Marvin David Marcus and Benjamin Nelson Moyls, Transformations on

tensor product spaces .................................. 1215

Charles Alan McCarthy, The nilpotent part of a spectral operator ........ 1223

Kotaro Oikawa, On a criterion for the weakness of an ideal boundary

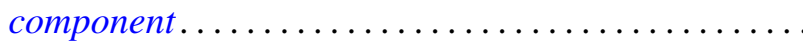

Barrett O'Neill, An algebraic criterion for immersion . . ............... 1239

Murray Harold Protter, Vibration of a nonhomogeneous membrane ... . . . . 1249

Victor Lenard Shapiro, Intrinsic operators in three-space . . . . . . . . . . . 1257

Morgan Ward, Tests for primality based on Sylvester's cyclotomic

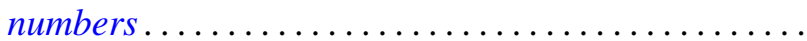

L. E. Ward, A fixed point theorem for chained spaces ....

Alfred B. Willcox, Šilov type $C$ algebras over a connected locally compact

abelian group..................................... 1279

Jacob Feldman, Correction to "Equivalence and perpendicularity of

Gaussian processes" ........................ 